\title{
Uso do propofol (2,6 diisopropilfenol) como inibidor da lesão tecidual na isquemia e reperfusão mesentérica. Estudo experimental em ratos $^{1}$
}

\author{
Carlos Henrique Marques dos Santos ${ }^{2}$ \\ Otoni Moreira Gomes ${ }^{3}$ \\ José Carlos Dorsa Vieira Pontes ${ }^{4}$ \\ Luciana Nakao Odashiro Miiji ${ }^{5}$ \\ Eric Iasuji Higa ${ }^{6}$
}

Santos CHM, Gomes OM, Pontes JCDV, Miiji LNO, Higa EI. Uso do propofol (2,6 diisopropilfenol) como inibidor da lesão tecidual na isquemia e reperfusão mesentérica. Estudo experimental em ratos. Acta Cir Bras [serial online] 2003 Jul-Ago;18(4). Disponível em URL: http://www.scielo.br/acb.

RESUMO - Objetivo: Avaliar os efeitos do propofol como inibidor da lesão tecidual na isquemia e reperfusão mesentérica em ratos.Métodos: Quarenta ratos Wistar foram submetidos à anestesia e laparotomia mediana. Obteve-se isquemia intestinal por falsa ligadura da artéria mesentérica cranial por trinta minutos. Após, reperfundiu-se por sessenta minutos. Metade dos animais receberam SF $0,9 \% 10 \mathrm{ml} / \mathrm{Kg} / \mathrm{hora}$ (grupo controle) por via intravenosa; a outra metade recebeu propofol $20 \mathrm{mg} /$ $\mathrm{Kg} /$ hora por mesma via (grupo propofol). Ao final, ressecou-se segmentos do intestino delgado para análise histológica. Avaliou-se os resultados pela classificação de CHIU et al ${ }^{80}$ e procedeu-se o tratamento estatístico.Resultados: No grupo controle encontrou-se 5 ratos com classificação grau 2; 8 com classificação grau 3; 3 com classificação grau 4 e 4 ratos com classificação grau 5. No grupo propofol encontrou-se 6 ratos com classificação grau 1; 11 com classificação grau 2 e 3 com classificação grau 3. Média de classificação 3,3 para o grupo controle e 1,85 para o grupo propofol. A variação dos dois grupos foi considerada estatisticamente significativa $(\mathrm{P}<0,05)$. Conclusão: O propofol minimiza a lesão tecidual em ratos submetidos a isquemia e reperfusão mesentérica.

DESCRITORES - Propofol. Isquemia. Reperfusão.

\section{Introdução}

A isquemia mesentérica aguda consiste na ausência ou diminuição acentuada do fluxo sangüíneo por comprometimento arterial, venoso ou da microcirculação intestinal.
A produção de radicais livres durante a fase de reperfusão, seguindo a isquemia é a principal causa de lesão tecidual. PARKS \& GRANGER ${ }^{1}$ demonstraram que três horas de isquemia seguidas por uma hora de reperfusão determinavam maior lesão na mucosa intestinal do que quatro horas de exclusiva isquemia.

1. Trabalho desenvolvido no Centro de Pesquisas do Hospital Regional de Mato Grosso do Sul.

2. Mestre em Medicina - Fundação Cardiovascular São Francisco de Assis.

3. Prof Titular de Clínica Cirúrgica da Universidade Federal de Minas Gerais (UFMG).

4. Prof Adjunto de Cirurgia Cardiovascular - Universidade Federal de Mato Grosso do Sul (UFMS).

5. Médica Patologista.

6. Acadêmico do Curso de Medicina - UFMS. 
O tratamento para a isquemia mesentérica, até pouco tempo atrás, restringia-se a ressecções intestinais associadas ou não a embolectomias. Tais procedimentos continuam sendo justificáveis em situações onde não mais é possível realizar a reperfusão, como na gangrena intestinal, por ser esta lesão irreversível. Entretanto, em muitas situações há lugar para um tratamento que bloqueie a seqüência de eventos do processo de isquemia e reperfusão, de modo a inibir ou minimizar seus efeitos danosos aos tecidos.

A obstrução abrupta do fluxo arterial pode ser decorrente de embolia ou trombose aguda, podendo ter por base um ateroma. Ocasionalmente, uma dissecção aórtica pode comprometer a origem dos troncos arteriais mesentéricos. Além do fator predisponente, a placa de ateroma, a trombose surge na dependência de fatores desencadeantes, tais como a hemoconcentração, a hipovolemia ou a redução do fluxo por causas gerais. A origem mais freqüente dos êmbolos é o coração, seja de trombo atrial, em pacientes com fibrilação, seja de um trombo decorrente de infarto recente do miocárdio, ou, ainda, de uma prótese valvar.

A oclusão venosa que leva a infarto intestinal devese a trombose aguda, que corresponde a pouco mais de $10 \%$ do total de casos de isquemia oclusiva aguda. A trombose venosa ocorre como complicação de condições clínicas variadas, tais como: infecções intraabdominais, estados de hipercoagulabilidade, congestão venosa local, estase e traumatismo operatório ou acidental.

Em algumas circunstâncias de infarto mesentérico não se observa obstáculo mecânico, recebendo por isso a denominação de não oclusivo. Esta denominação, amplamente utilizada, não é de todo correta, pois, no grupo etário atingido, são relativamente comuns as estenoses ateromatosas dos troncos arteriais, como também as lesões degenerativas das pequenas artérias, as quais, muito provavelmente, contribuem para a isquemia. A causa mais comum é o baixo débito cardíaco, decorrente de toxemia, insuficiência cardíaca congestiva, arritmias cardíacas, infarto agudo do miocárdio, hipovolemia profunda e, em certas circunstâncias, a própria cirurgia cardíaca com circulação extracorpórea.

A terapêutica pode também contribuir para essa situação. Assim, os vasopressores utilizados no tratamento de estados de choque e os digitálicos, são substâncias capazes de induzir vasoconstricção mesentérica, desencadeando ou agravando a isquemia tecidual.
Outra situação que vem ganhando interesse cada vez maior nos últimos anos é o transplante de órgãos, incluindo-se aqui o transplante de intestino delgado. $\mathrm{O}$ órgão a ser transplantado permanece tempo variável em regime de isquemia, sendo posteriormente submetido à reperfusão, ficando, portanto, suscetível à lesão tecidual decorrente deste processo.

Visando evitar ou minimizar os efeitos maléficos da isquemia e reperfusão mesentérica em situações como as acima referidas, diversas substâncias têm sido estudadas. Algumas destas substâncias possuem efeito anti-oxidante capaz de prevenir as lesões de reperfusão tecidual. Dessa forma, a busca de outros fármacos capazes de reduzir os efeitos deletérios da reperfusão, tem sido objeto de estudo de vários pesquisadores.

Alguns autores ${ }^{2-10}$ têm utilizado o propofol (2,6 diisopropilfenol) na síndrome de isquemia e reperfusão devido a sua ação de inibir a peroxidação lipídica. Tais autores obtiveram sucesso na utilização do propofol como agente anti-oxidante em cirurgias neurológicas na prevenção dos efeitos deletérios do processo de isquemia e reperfusão cerebral. Não há relatos na literatura médica consultada, no entanto, de que este fármaco tenha sido utilizado com tal propósito na isquemia e reperfusão mesentérica.

Assim, a presente investigação tem por objetivo estudar o propofol, uma substância de reconhecido efeito anti-oxidante, na prevenção ou diminuição dos efeitos nocivos do processo de isquemia e reperfusão mesentérica.

\section{Métodos}

Foram estudados quarenta ratos (Rattus norvegicus albinus, Rodentia, Mammalia), da linhagem Wistar, machos, adultos, com peso variando de 270 a 350 gramas, com média de 305 gramas, provenientes do Biotério da Universidade Federal de Mato Grosso do Sul.

Os animais foram distribuídos nos seguintes grupos:

- Grupo I - Controle:

Vinte ratos submetidos ao procedimento cirúrgico recebendo SF $0,9 \%$ por via intravenosa.

\section{- Grupo II - Propofol:}

Vinte ratos submetidos ao procedimento cirúrgico recebendo propofol por via intravenosa.Os animais foram anestesiados inicialmente com éter etílico a 
97 \% por via inalatória em campânula fechada. Após a perda postural, foi feita injeção intraperitoneal de citrato de sufentanila na dose de $12 \mathrm{mcg} / \mathrm{kg}$ e tiopental sódico na dose de $18 \mathrm{mg} / \mathrm{kg}$.

Os ratos foram fixados à mesa cirúrgica em decúbito dorsal com os quatro membros em abdução. Em seguida foi realizada cervicotomia e dissecção da veia jugular interna, na qual foi introduzido cateter plástico número $25 \mathrm{G}$, fixado por ligadura com fio de algodão 000. Foi então iniciada a infusão intravenosa de SF 0,9\% (no grupo controle) $10 \mathrm{ml} / \mathrm{Kg} / \mathrm{hora}$ e propofol (no grupo propofol) na dose de $20 \mathrm{mg} / \mathrm{kg} /$ hora. O propofol foi diluído em SF $0,9 \%$ na proporção de 1:5, formando uma solução de $1 \mathrm{ml}=0,5 \mathrm{mg}$, de forma a haver correspondência entre os volumes infundidos de SF 0,9\% (grupo I) e propofol (grupo II). A infusão das soluções foi feita por meio de bomba de infusão.

Em seguida, realizou-se laparotomia longitudinal mediana de aproximadamente 4 centímetros, exteriorização das alças intestinais, identificação e dissecção da artéria mesentérica cranial, a qual foi ocluída por falsa ligadura com fio de algodão 000, mantido tracionado por pinça hemostática reta que permaneceu durante trinta minutos (fase de isquemia). Durante esta fase, as alças intestinais foram reposicionadas na cavidade abdominal e a ferida operatória foi coberta com gaze umedecida com SF 0,9\% morna.

Após trinta minutos de isquemia a falsa ligadura foi retirada, momento em que se observou as alças intestinais com coloração vinhosa e edemaciadas. Novamente as alças foram reposicionadas na cavidade peritoneal por mais sessenta minutos (fase de reperfusão).

Completada esta fase, foi ressecado um segmento de aproximadamente $1 \mathrm{~cm}$ do íleo, $10 \mathrm{~cm}$ proximal à transição ileocecal, o qual foi colocado em solução de formol a $10 \%$ para posterior análise histológica.

Realizou-se monitorização da oximetria por sensor colocado na pata traseira dos animais, mantendo-se a saturação arterial de oxigênio acima de $94 \%$ durante todo o procedimento, com ventilação espontânea em ar ambiente.

A eutanásia foi feita por aprofundamento do plano anestésico.

Os segmentos ressecados, após fixação em solução de formaldeído a $10 \%$, foram submetidos a processamento histológico por doze horas no histotécnico automático. Após o processamento foram embebidos em parafina e submetidos a cortes histológicos em macrométrio a cada $4 \mathrm{mcm}$.

As lâminas foram coradas por hematoxilina-eosina e analisadas à microscopia óptica pelo patologista, sem o prévio conhecimento sobre o grupo pertencente de cada rato, e foram classificados de acordo com o grau de lesão tecidual segundo CHIU, McARDLE, BROWN, SCOTT e GURD ${ }^{11}$ :

1 Grau 0: mucosa sem alterações

1 Grau 1: vilosidades bem constituídas, sem lise celular ou processo inflamatório, porém, com formação do espaço subepitelial de Grunhagen

1 Grau 2: presença de lises celulares, formação do espaço subepitelial de Grunhagen e espaçamento aumentado entre as vilosidades

1 Grau 3: destruição da porção livre das vilosidades, presença de capilares dilatados e de células inflamatórias

1 Grau 4: destruição estrutural das vilosidades, havendo apenas esboço de algumas, formadas por células inflamatórias e material necrótico, com hemorragia e ulceração glandular basal

1 Grau 5: destruição de toda túnica mucosa, não mais sendo observado qualquer estrutura glandular, mas apenas material amorfo depositado sobre a tela submucosa.

Os resultados obtidos foram submetidos a tratamento estatístico, aplicando-se o Teste " $t$ " de Student para comparações pareadas, sendo estabelecido o nível de significância de $95 \%$. Foi utilizado o programa EXCEL 7.0, da Microsoft.

\section{Resultados}

O programa de pesquisa foi cumprido em quarenta ratos. Os resultados da análise histológica encontramse na Tabela 1.

Após a análise histológica pela microscopia óptica observou-se que o grupo controle apresentou classificação grau 2 de CHIU em 5 ratos, grau 3 em 8 ratos, grau 4 em 3 ratos, e, em 4 ratos classificação grau 5, conforme se observa na figura 1 . Não se obteve classificação graus 0 e 1 neste grupo.

No grupo propofol não se obteve classificação graus 0,4 e 5 em nenhum rato. Em 6 ratos, obteve-se classificação grau 1, em 11, classificação grau 2, e, em 3 ratos, classificação grau 3 (Figura 1). 
Santos C H M e col.

TABELA 1 - Resultado da análise histológica das mucosas dos segmentos intestinais dos grupos de ratos tratados ou não com propofol e submetidos a 30 minutos de isquemia e 60 minutos de reperfusão.

\begin{tabular}{ccc}
\hline RATOS & CONTROLE & PROPOFOL \\
\hline 01 & 3 & 2 \\
02 & 3 & 2 \\
03 & 2 & 2 \\
04 & 3 & 2 \\
05 & 2 & 3 \\
06 & 3 & 2 \\
07 & 2 & 1 \\
08 & 3 & 1 \\
09 & 3 & 3 \\
10 & 4 & 2 \\
11 & 3 & 2 \\
12 & 3 & 1 \\
13 & 4 & 1 \\
14 & 4 & 2 \\
15 & 2 & 3 \\
16 & 5 & 2 \\
17 & 2 & 2 \\
18 & 5 & 1 \\
19 & 5 & 2 \\
20 & 5 & 1 \\
\hline MÉDIA & 3,3 & 1,85 \\
DP & 1,080935 & 0,67082 \\
\hline
\end{tabular}

Fonte: Resultado da análise histológica desta pesquisa segundo a classificação de CHIU et al ${ }^{11}$.

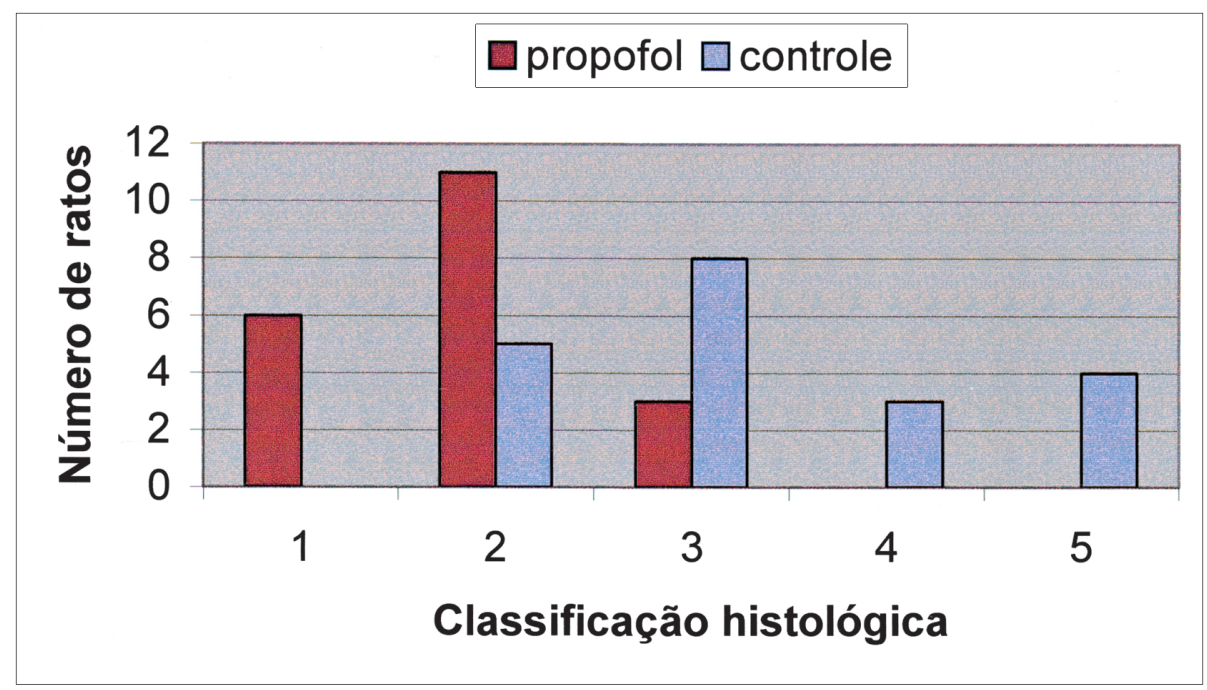

FIGURA 1 - Gráfico comparativo da análise histológica das mucosas intestinais dos grupos de ratos tratados ou não com propofol submetidos a 30 minutos de isquemia e 60 minutos de reperfusão. 


\section{Discussão}

O propofol, objeto do presente estudo, tem apresentado efeitos positivos na prevenção da lesão tecidual frente à isquemia e reperfusão ${ }^{2-10}$.

O propofol tem estrutura química similar aos núcleos ativos do tocoferol e do butilidroxitolueno, antioxidantes endógenos. Estas moléculas aderem às membranas celulares ou aos fosfolípides para formar radicais livres, e inibem a peroxidação lipídica por reagirem com os radicais lipídicos peroxil para formar o não reativo radical fenoxil. O propofol pode agir por meio de mecanismo similar. Seus efeitos anti-oxidantes resultariam da inibição da peroxidação lipídica pela formação de radicais livres relativamente pouco reativos que quebrariam a cadeia de formação de outros radicais potencialmente danosos às membranas celulares ${ }^{6}$.

No presente estudo observou-se um efeito benéfico do propofol em relação ao grupo controle em vigência de isquemia e reperfusão mesentérica. As médias de classificação histológica foram de 3,3 e 1,85 para os grupos controle e propofol, respectivamente.

A classificação histológica de CHIU et al ${ }^{11}$, descrita em 1970, tornou-se clássica na avaliação de lesão tecidual intestinal por isquemia e reperfusão, sendo utilizada por diversos autores para esta finalidade.

SHAN, BAIXIANG, CATY e TJOTA ${ }^{12}$ analisaram a regeneração da mucosa intestinal em ratos após períodos diferentes de isquemia e reperfusão e observaram que nos espécimes onde havia destruição total dos vilos (correspondendo à classe 4 de CHIU et al ${ }^{11}$ ), após dois dias os vilos estavam escassos. Por outro lado, os espécimes nos quais na avaliação inicial só havia destruição do topo das vilosidades, dois dias após apresentavam regeneração total. Também BIONDO-SIMÕES, GRECA, IOSHI, TAWIL, MENINI e RAMPAZZO ${ }^{13}$ consideraram como lesões irreversíveis aquelas em que houve destruição total dos vilos e reversíveis até a fase anterior onde houve lesão incompleta das vilosidades.

Por estas observações, poder-se-ia dizer que todos os ratos do grupo propofol do presente estudo teríam apenas lesões reversíveis, visto que o máximo grau de lesão observado à histologia foi 3.

KAÇMAZ, OZTURK, KARAAYVAZ, GUVEN e DURAK $^{14}$, estudando o Alopurinol como anti-oxidante na isquemia e reperfusão intestinal, obtiveram média 4,6 na classificação histológica de CHIU et al ${ }^{11}$ para o grupo controle e 1,8 para o grupo Alopurinol. Estes autores utilizaram 5 ratos Sprague-Dawley em cada grupo e mantiveram os animais por quarenta minutos de isquemia seguidos por quarenta minutos de reperfusão.

No presente estudo optou-se por trinta e sessenta minutos de isquemia e reperfusão, respectivamente, com base no estudo realizado por BOROS, TAKAICHI e HATANAKA ${ }^{15}$, no qual analisaram a relação entre tempo de isquemia e reperfusão intestinal em ratos e a lesão tecidual. Estes autores concluíram que com trinta minutos de oclusão arterial a isquemia passa a ser severa, ocorrendo desintegração da microcirculação da extremidade dos vilos. Concluíram ainda que o componente de reperfusão teria pequeno significado durante os estágios tardios de isquemia, mas poderia ser muito importante na patogênese da lesão mucosa seguindo formas precoces de oclusão completa da circulação mesentérica.

Outros autores ${ }^{16-19}$ também utilizaram os mesmos períodos de isquemia e reperfusão mesentérica que os utilizados no presente estudo.

ONEN, DEVECI, INALOZ, ISIK e KILINC ${ }^{20}$, obtiveram média de classificação histológica 4,3 para o grupo controle e 2 para o grupo que recebeu extrato de Ginkgo-biloba como agente anti-oxidante antes da laparotomia. Porém, apesar de terem deixado os ratos sob isquemia mesentérica por trinta minutos, o período de reperfusão foi de apenas vinte minutos. YONGMING, YAN, YE, ZHIGUO e ZHIYONG ${ }^{21}$ observaram que ratos submetidos a isquemia por oclusão da artéria mesentérica superior por quarenta e cinco minutos e posteriormente reperfundidos, apresentaram níveis de fator de necrose tumoral plasmáticos duas vezes maior após 2 horas de reperfusão em comparação ao grupo com trinta minutos. $\mathrm{O}$ fator de necrose tumoral foi utilizado para avaliar lesão tecidual pósisquemia e reperfusão. Desta forma, é possível que com período de reperfusão maior os resultados de ONEN et al ${ }^{20}$ não fossem tão bons, dificultando a comparação com o presente estudo.

BIONDO-SIMÕES et al ${ }^{13} \mathrm{em}$ trabalho no qual utilizaram método bastante semelhante ao deste estudo, porém, com reperfusão por trinta minutos apenas, estudaram o uso de eritromicina administrada previamente à isquemia e reperfusão mesentérica em ratos e observaram não haver benefício utilizando tal substância em relação ao grupo controle.

Estudando a L-arginina como protetor da lesão tecidual na isquemia e reperfusão intestinal, WARD, LAWSON, GALLAGHER, CONNER e SHEADONOHUE ${ }^{16}$ obtiveram classificação histológica média de 3,9 para o grupo controle, 2,2 com a administração de L-arginina antes da isquemia e 3,1 antes da reperfusão, sugerindo haver potencial benefício de tal 
substância na prevenção da injúria tecidual. Também GUO, CHAN, FUNG, CHAN e TAM ${ }^{22}$ estudaram a Larginina e observaram um aumento na produção de óxido nítrico e conseqüente redução da injúria pósisquemia e reperfusão intestinal. De maneira oposta, KHANNA, ROSSMAN, FUNG e CATY ${ }^{23}$ afirmaram que o óxido nítrico apresenta fundamental participação na isquemia e reperfusão, estando o aumento de seus níveis relacionados a proporcional aumento na injúria tecidual. DURAKBASA, DAGLI, MOUNI et al ${ }^{18}$ também afirmaram que o níveis elevados de óxido nítrico estão associados a injúria tecidual na isquemia e reperfusão. Com tal controvérsia, apesar de indícios de proteção tecidual oferecida pela L-arginina, fica difícil conhecer seu real mecanismo de ação, necessitando outros estudos mais aprofundados para o esclarecimento a respeito da participação do óxido nítrico na isquemia e reperfusão tecidual.

LEE, McCAULEY, KONG e HALL ${ }^{24}$ não observaram diferença na classificação histológica entre o grupo controle e o grupo que recebeu glicina previamente à isquemia em seu estudo. Entretanto, há uma diferença importante no método em comparação com o presente estudo, visto que LEE et al ${ }^{24}$ realizaram oclusão aórtica por trinta minutos em nível de artéria celíaca seguido por trinta minutos de reperfusão.

Em nosso meio, MACARENCO, TAKAHAGI, BARDELLA, SEQUEIRA e YOSHIDA ${ }^{25}$, utilizaram também a classificação histológica de CHIU et al ${ }^{11}$ na análise de seus resultados em trabalho comparando a ação do extrato de Ginkgo-biloba e amido hidroxietílico hipertônico na atenuação de alterações decorrentes da isquemia e reperfusão de órgãos esplâncnicos em ratos. Não houve diferença estatística entre os grupos utilizando os fármacos e o grupo controle. O período de isquemia foi semelhante ao deste estudo, porém, a reperfusão foi feita durante noventa minutos.

Outros métodos de avaliação da lesão tecidual tem sido utilizados em estudos experimentais, além da análise histológica. O principal objetivo é encontrar uma forma pouco invasiva de auxiliar no diagnóstico precoce da síndrome de isquemia e reperfusão mesentérica.

Em nosso meio, BRITO, ARAÚJO, ACÁCIO, ACÁCIO e REIS ${ }^{26}$ compararam a avaliação histológica e o estudo colorimétrico usando sal tetrazólico em ratos submetidos a isquemia e reperfusão mesentérica, mostrando haver equivalência quanto à sensibilidade dos métodos. Entretanto, o método não-histológico mais utilizado é a dosagem dos níveis teciduais e plasmáticos do malondialdeído, um radical lipídico que se encontra aumentado durante a reperfusão.
LO, CHEN, CHEN et al ${ }^{17}$ relataram que a aminoguanidina foi capaz de reduzir os níveis de peroxidação lipídica intestinal e hepática realizando-se isquemia ileal por oclusão da artéria mesentérica superior durante trinta minutos e reperfusão também por trinta minutos. Os níveis intestinais e pulmonares de malondialdeído foram menores no grupo em que se utilizou aminoguanidina que no grupo controle.

HAMMERMAN, GOLDSCMIDT, CAPLAN et $\mathrm{al}^{27}$ em estudo experimental em ratos, analisaram os efeitos da pentoxifilina como mediadora da inibição da xantina-oxidase na isquemia mesentérica, realizando oclusão da artéria mesentérica superior por quarenta e cinco minutos e reperfusão de trinta e sessenta minutos, observando níveis significativamente maiores de xantina-oxidase e de xantina-desidrogenase no grupo controle em relação aos grupos utilizando a pentoxifilina. Entretanto, tais substâncias encontram-se aumentadas na fase de isquemia, e, embora seja provável que desencadeiem reações que poderiam levar à lesão tecidual, não são marcadores de lesão de reperfusão.

KLOTZ et al ${ }^{28}$ realizaram estudo prospectivo nãorandomizado em pacientes em pós-operatório de cirurgias cardíacas ( revascularização miocárdica, troca de válvula aórtica e transplante cardíaco) com quadro clínico compatível com isquemia mesentérica. Submeteram vinte pacientes à arteriografia mesentérica, dos quais quatorze tiveram o diagnóstico de isquemia mesentérica não-oclusiva. Estes quatorze pacientes receberam papaverina por cateter posicionado na origem da artéria mesentérica superior. Nove tiveram melhora e 5 necessitaram tratamento operatório com ressecção intestinal segmentar. Destes 5, 3 foram a óbito. A papaverina é um vasodilatador potente já utilizado em humanos sem efeitos colaterais ou complicações, segundo estes autores. Entretanto, 5 pacientes (35\%) não tiveram boa resposta com seu emprego e necessitaram intervenção cirúrgica.

Assim, observa-se que alguns fármacos demonstraram capacidade de inibir a lesão tecidual na isquemia e reperfusão mesentérica. Entretanto, é necessário que novos estudos sejam feitos com as substâncias que até aqui apresentaram resultados positivos, no sentido de se avaliar as doses ideais, as possíveis interações medicamentosas e o momento ideal de início e término do tratamento. Ao mesmo tempo, faz-se necessário que novas substâncias sejam estudadas visando encontrarse outras possibilidades terapêuticas. Com este propósito foi que analisou-se no presente estudo os efeitos do propofol na prevenção das lesões de reperfusão, observando-se que tal fármaco foi capaz de minimizar a lesão tecidual após isquemia e reperfusão mesentérica em ratos. 


\section{Conclusão}

O propofol na dose de $20 \mathrm{mg} / \mathrm{kg} / \mathrm{hora}$ minimiza a lesão tecidual em ratos submetidos à isquemia e reperfusão mesentérica.

\section{Referências}

1. Parks DA, Granger DN. Contributions of ischemia and reperfusion to mucosal lesion formation. Am J Physiol 1986; 250: 749-53

2. Wolf A, Weir P, Segar P, Stone J, Shield J. Impaired fatty acid oxidation in propofol infusion syndrome. Lancet 2001; 357: 606-07.

3. Oda Y, Hamaoka N, Hiroi T, Irmaoka S, Hase I, Tanaka K, Funae Y, Ishizaki T, Asada A. Involvement of human liver cytocrome P4502B6 in the metabolism of propofol. Br J Clin Pharmacol 2001; 51: 281-85.

4. Lee Y, Chumg C, OH YS. Effectiveness of propofol pretreatment on the extent of deranged cerebral mitochondrial oxidative enzyme system after incomplete forebrain ischemia/ reperfusion in rats. J Korean Med Sci 2000; 15(6): 627-30.

5. Yano T, Nakayama R, Ushijima K. Intracerebroventricular propofol is neuroprotective against transient global ischemia in rats: extracellular glutamate level is not a major determinant. Brain Res 2000; 883: 69-76.

6. Hamaguchi S, Mishio M, Okuda Y, Kitajima T. Propofol and lipid peroxidation. Can J Anaesth 2000; 47: 1025-30.

7. Bao YP, Williamson G, Tew D, Plumb GW, Lambert N, Jones JG, Menon DK. Antioxidant effects of propofol in human hepatic microsomes: concentration effects and clinical relevance. Br J Anesth 1998; 81(4): 584-9.

8. De La Cruz JP, Sedeno G, Carmona JA, De La Cuesta FS. The in vitro effects of propofol on tissular oxidative stress in the rat. Anesth Analg 1998; 87: 1141-6.

9. Hans P, Deby-Dupont G, Deby C, Pieron F, Verbesselt R, Franssen C, Lamy M. Increase in antioxidant capacity of plasma during propofol anesthesia. J Neurosurg Anesth 1997; 9(3): 234-6.

10. Ansley DM, Lee J, Godin DV, Garnett ME, Qayumi AK. Propofol enhances red cell antioxidant capacity in swine and humans. Can J Anaesth 1998; 45(3): 233-9.

11. Chiu CJ, McArdle AH, Brown R, Scott HJ, Gurd FN. Intestinal mucosal lesion in low-flow states. Arch Surg 1970; 101: 478-83.

12. Shan Z, Baixiang J, Caty MG, Tjota A. Mucosa cell regeneration following intestinal ischemia/reperfusion injury in rats. Chin Med J 1997; 110(5): 338-40.

13. Biondo-Simões MLP, Greca FH, Ioshi S, Tawil IIE, Menini CM, Rampazzo JC. The influence of antibiotics on intestinal ischemia and reperfusion: experimental study in rats. Acta Cir Bras 2000; 15(3): 83-7.

14. Kaçmaz M, Ozturk HS, Karaayvaz M, Guven C, Durak I. Enzymatic antioxidant defence mechanism in rat intestinal tissue is changed after ischemia-reperfusion. Effects of an allopurinol plus antioxidant combination. Can J Surg 1999; 42: 427-31.
15. Boros M, Takaichi S, Hatanaka K. Ischemia time dependency of reperfusion injury following complete arterial occlusion of the rat small intestine. Transpl Proc 1995; 27(5): 2789-90.

16. Ward DT, Lawson AS, Gallagher CM, Conner WC, SheaDonohue T. Sustained nitric oxide production via L-arginine administration ameliorates effects of intestinal ischemiareperfusion. J Surg Res 2000; 89: 13-9.

17. Lo CC, Chen JC, Chen HM, Shyr MH, Lau YT, Lin JN, Chen MF. Aminoguanidine attenuates hemodynamic and microcirculatory derangement in rat intestinal ischemia and reperfusion. J Trauma 1999; 47(6): 1108-13.

18. Durakbasa ÇU, Dagli TE, Mouni H, Haklar G, Bilsel AS, Yuksel M, Aktan AO. Nitric oxide and endothelin relationship in intestinal ischemia/reperfusio injury. Prostaglandins Leukot Essent Fatty Acids 1998; 59(6): 379-83.

19. Zhang Y, Wu YX, Hao YB, Dun Y, Yang SP. Role of endogenous opioid peptides in protection of ischemic preconditioning in rat small intestine. Life Sci 2001; 68: 1013-19.

20. Onen A, Deveci E, Inaloz SS, Isik B, Kilinc M. Histopathological assesment of the prophylactic effect of gingko-biloba extract on intestinal ischemia-reperfusion injury. Acta Gastroenterol Belg 1999; 62: 386-9.

21. Yongming Y, Yan Y, Ye W, Zhiguo S, Zhiyong S. The role of gut as a cytokine-generating organ in remote organ dysfunction after intestinal ischemia and reperfusion. Chin Med J 1998; 111(6): 514-8.

22. Guo WH, Chan KL, Fung PPCW, Chan KW, Tam PKH. Nitric oxide protects segmental intestinal grafts from ischemia and reperfusion injury. Transpl Proc 2000; 32: 1297-8.

23. Khanna A, Rossman JE, Fung HL, Caty MG. Attenuated nitric oxide synthase activity and protein expression accompany intestinal ischemia/reperfusion injury in rats. Biochem Biophys Res Commun 2000; 269: 160-4.

24. Lee MA, McCauley RD, Kong SE, Hall JC. Pretreatment with glycine reduces the severity of warm intestinal ischemicreperfusion injury in the rat. Ann Plastic Surg 2001; 46(3): 320-6.

25. Macarenco RSS, Takahagi RU, Bardella LC, Sequeira JL, Yoshida WB. Estudo da ação do extrato de Gingko biloba e amido hidroxietílico hipertônico na atenuação de alterações decorrentes de isquemia e reperfusão de órgãos esplâncnicos em ratos. Acta Cir Bras jul/ago/set 2001; 16(3): 139-45.

26. Brito MVH, Araújo M, Acácio GJS, Acácio GJS, Reis JMC. Lesão intestinal após isquemia-reperfusão: estudo comparativo usando sal tetrazólico (MTT) e Histologia. Acta Cir Bras jan/ fev/mar 2001; 16(1): 26-31.

27. Hammerman C, Goldschmidt D, Caplan MS, Kaplan M, Schimmel MS, Eidelman AI, Branski D, Hochman A. Amelioration of ischemia-reperfusion injury in rat intestine by pentoxifylline-mediated inhbition of xanthine oxidase. J Pediatr Gastroenterol Nutr 1999; 29: 69-74.

28. Klotz S, Vestring T, Rotker J, Schmidt C, Scheld HH, Schmid C. Diagnosis and treatment of nonocclusive mesenteric ischaemia after open heart surgery. Ann Thorac Surg 2001; 72(5): 1583-6. 
Santos CHM, Gomes OM, Pontes JCDV, Miiji LNO, Higa EI. Use of propofol (2,6 diisopropilphenol) as inhibitor of tissue lesion in mesenteric ischemia and reperfusion: experimental study in rats. Acta Cir Bras [serial online] 2003 Jul-Aug;18(4). Available from URL: http://www.scielo.br/acb.

ABSTRACT - Purpose: Evaluate the effects of propofol as inhibitor of tissue lesion in mesenteric ischemia and reperfusion in rats. Methods: Forty rats Wistar were submitted to anesthesia and middle laparotomy. Intestinal ischemia was obtained by false ligature of cranial mesenteric artery by thirty minutes. Then, the tissue was reperfused for sixty minutes. The half of the animals received physiologic solution $0,9 \% 10 \mathrm{ml} / \mathrm{kg} / \mathrm{h}$ (control group) by intravenous infusion; the other half received propofol $20 \mathrm{mg} / \mathrm{kg} / \mathrm{h}$ by same way (propofol group). At the end, a segment of intestine was taken for histologycal analysis. The results were analyzed in agreement with CHIU et al ${ }^{80}$ classification and the statistical analysis was done. Results: In the control group was founded 5 rats with classification grade 2;8 with classification grade 3; 3 with classification grade 4 and 4 rats with grade. In the propofol group was found 6 rats with classification grade $1 ; 11$ with classification grade 2 and 3 with classification grade 3 . The classification media was 3,3 for control group and 1,85 for the propofol group. The variation in the two groups was considered statistically significant $(\mathrm{P}<0,05)$. Conclusion: The propofol minimize the tissue lesion in mesenteric ischemia and reperfusion in rats.

KEY WORDS - Propofol. Ischemia. Reperfusion.

Conflito de interesse: nenhum

Fonte de financiamento: nenhuma

Correspondência:

Carlos Henrique Marques dos Santos

R. Cora Coralina, 91/casa 7

79040-510 Campo Grande - MS

Data do recebimento: 08/04/2003

Data da revisão: 18/04/2003

Data da aprovação: 03/05/2003 